\title{
Estratégia cirúrgica para tratamento de adenoma pleomórfico de grande tamanho: relato de caso
}

Surgical strategy for treatment of large pleomorphic adenoma: case report

Estrategia quirúrgica para el tratamiento del adenoma pleomórfico grande: reporte de caso

\author{
Breno dos Reis FERNANDES ${ }^{1}$ \\ Darah Ligia MARCHIRORI ${ }^{2}$ \\ Oswaldo BELLOTI NETO ${ }^{3}$ \\ Patrese Pereira DE BELLA ${ }^{3}$ \\ Gabriel MULINARI-SANTOS ${ }^{1}$ \\ Raphael CENCI ${ }^{3}$ \\ Luana Carolina SASSI \\ Juliana Zorzi COLÉTE ${ }^{4}$ \\ ${ }^{I}$ Faculdade de Odontologia de Araçatuba, UNESP-Univ. Estadual Paulista, Araçatuba-SP, Brasil \\ ${ }^{2}$ Universidade Alto Vale do Rio do Peixe, Cacador-SC, Santa Catarina, Brasil \\ ${ }^{3}$ Hospital das Clínicas de Teresópolis - RJ, UNESP-Univ. Estadual Paulista, Teresópolis-, Brasil \\ ${ }^{4}$ Universidade Estadual do Norte do Paraná-PR, UENP, Jacarézinho-PR, Brasil
}

\section{Resumo}

As neoplasias da glândula parótida constituem um grupo heterogêneo com mais de 30 tipos histológicos definidos, sendo o adenoma pleomórfico o tumor benigno mais comum. Neste artigo é relatado um caso clínico onde o tumor apresentou evolução de um ano, sem sintomatologia associada, gerando assimetria facial ao paciente, sendo realizado tratamento cirúrgico conservador apenas por enucleação da lesão, visando reduzir cirurgias maiores como a parotidectomias parciais ou totais. Após acompanhamento de 5 anos o paciente não apresenta sequelas do tratamento cirúrgico, nem sinal de recorrência da lesão.

Descritores: Adenoma Pleomorfo; Glândula Parótida; Relatos de Casos.

\section{Abstract}

The neoplasias of the parotid gland constitute a heterogeneous group with more than 30 defined histological types, and the pleomorphic adenoma is the most common benign tumor. In this article, a clinical case was reported where the tumor presented evolution of one year, without associated symptomatology, generating facial asymmetry to the patient, being carried out conservative surgical treatment only by enucleation of the lesion, aiming to reduce major surgeries such as partial or total parotidectomies. After 5 years of follow-up, the patient did not present sequels of the surgical treatment, nor did the patient have recurrence of the lesion.

Descriptors: Adenoma, Pleomorphic; Parotid Gland; Case Reports.

\section{Resumen}

Las neoplasias de la glándula parótida constituyen un grupo heterogéneo con más de 30 tipos histológicos definidos, siendo el adenoma pleomórfico el tumor benigno más común. En este artículo se relata un caso clínico donde el tumor presentó evolución de un año, sin sintomatología asociada, generando asimetría facial al paciente, siendo realizado tratamiento quirúrgico conservador sólo por enucleación de la lesión, buscando reducir las cirugías mayores como la parotidectomías parciales o totales. Después del seguimiento de 5 años el paciente no presenta secuelas del tratamiento quirúrgico, ni señal de recurrencia de la lesión.

Descriptores: Adenoma Pleomórfico; Glándula Parótida; Informes de Casos.

\section{INTRODUÇÃO}

Atualmente existem mais de 30 tipos de neoplasias da glândula parótida, de acordo com a classificação da Organização Mundial de Saúde (OMS), publicada em $1991^{1}$.

O adenoma pleomórfico é a neoplasia benigna mais comum da glândula parótida, representando cerca de 60 a $70 \%$, e com maior incidência a partir da $4 \mathrm{a}$ até a $6 \mathrm{a}$ década de vida ${ }^{2,3}$. Quando essa lesão afeta glândulas salivares menores, o local de acometimento mais freqüente é a região de palato duro, seguida do lábio superior, língua, assoalho de boca e região retromolar ${ }^{4}$.

Clinicamente, apresentam-se como lesões solitárias, ovóides, de margens bem delimitadas. A lesão é móvel, exceto quando ocorre no palato, apresentando crescimento lento e assintomático, suas dimensões podem variar de poucos milímetros a vários centímetros, com ligeira predominância no gênero feminino $0^{5,6}$. Na glândula parótida o adenoma pleomórfico acometem (90\%) dos casos o lobo superficial da glândula, sendo $80 \%$ na sua porção inferior ${ }^{3}$.

Microscopicamente, a variedade de tipos celulares é a principal característica do adenoma pleomórfico, não apenas entre diferentes tumores, mas também em diferentes partes de um mesmo tumor. É composto por elementos epiteliais, mioepiteliais e mesenquimais, envoltos em um estroma de natureza mixóide, condróide ou mesmo osteóide ${ }^{7}$.

O diagnóstico diferencial de adenoma pleomórfico inclui o adenoma monomórfico, adenoma oxifítico, cisto adenoma linfomatoso papilar, mucocele, fibroma, lipoma, além de neoplasias malignas como o carcinoma mucoepidermóide, adenocarcinoma, carcinoma adenomatóide cístico e carcinoma de células $\operatorname{acinosas}^{8}$. A conduta inicial como em qualquer lesão com indefinição clínica é a realização de uma biópsia, que dependendo da localização do tumor será incisional ou excisional ${ }^{9}$.

\section{CASO CLÍNICO}

Paciente do sexo masculino, 55 anos de idade, leucodema, compareceu no ambulatório de Cirurgia e Traumatologia Buco Maxilo Facial do Hospital das Clínicas de Teresópolis/RJ queixando-se de um aumento de volume do lado direito do seu rosto, que estava evoluindo há cerca de um ano. Ao exame físico apresentou aumento de volume 
assintomático, com evolução de 1 ano em região de glândula parótida direita (Figura1). Durante a palpação da lesão foi encontrado uma consistência flutuante, móvel, bem delimitada e indolor. Diante os achados clínicos a hipótese de diagnóstico foi de adenoma pleomórfico.

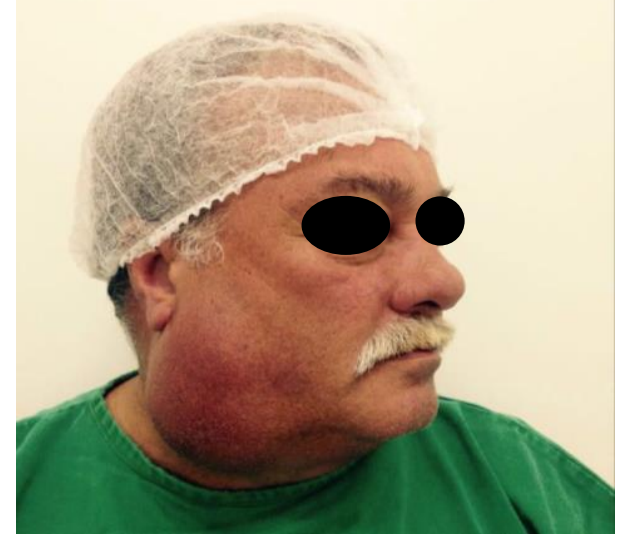

Figura 1: Aumento de volume em região de glândula parótida direita bem delimitada (vista lateral).

O paciente foi submetido a anestesia geral com entubação nasal, sendo realizado um acesso submandibular para exposição da lesão que encontrava- se em intimidade com a glândula parótida (Figura 2).

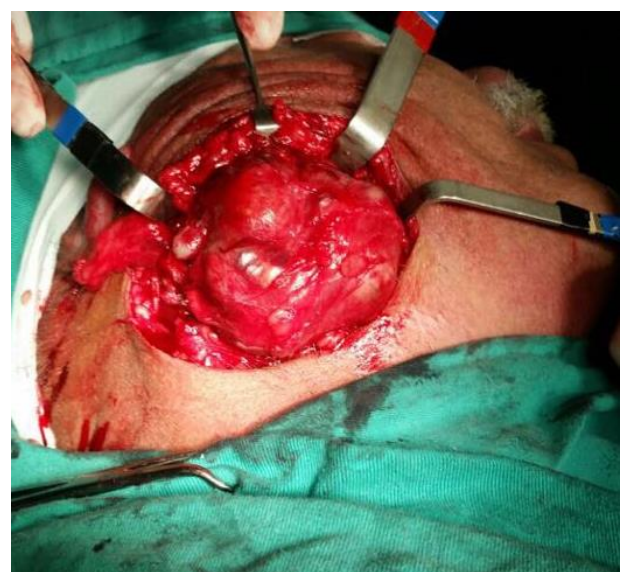

Figura 2: Acesso submandibular e visualização da lesão em região de glândula parótida.

Após dissecção extracapsular cuidadosa, o tumor pode ser enucleado sem o rompimento da cápsula (Figuras 3 e 4). Os tecidos profundos foram suturados com Vycril 4.0 e a pele com Nylon 5.0, e realizou-se o curativo compressivo. No segundo dia após o procedimento o mesmo recebeu alta para acompanhamento ambulatorial, com prescrição de: ibuprofeno $600 \mathrm{mg}$ a cada 08 duras por 03 dias e dipirona 500mg a cada 6 horas por 05 dias, além de amoxicilina 500mg a cada 8 horas por 7 dias. No sétimo dia de pós-operatório foi realizado a remoção de sutura, e mantido acompanhamento ambulatorial semanal por 8 semanas, mensal por 3 meses, e após apenas semestral. Atualmente o paciente apresenta 03 anos de pós-operatório sem intercorrências (Figura 5).

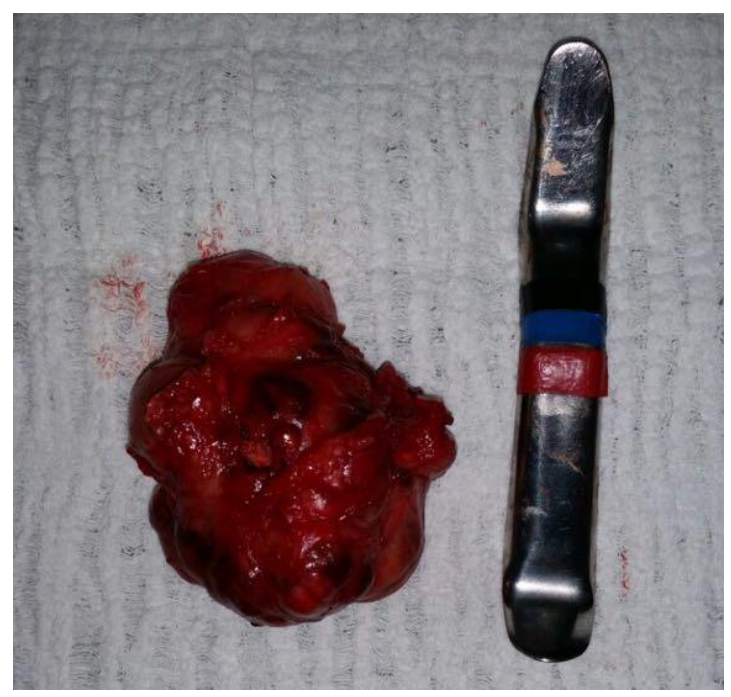

Figura 3: Lesão enucleada.

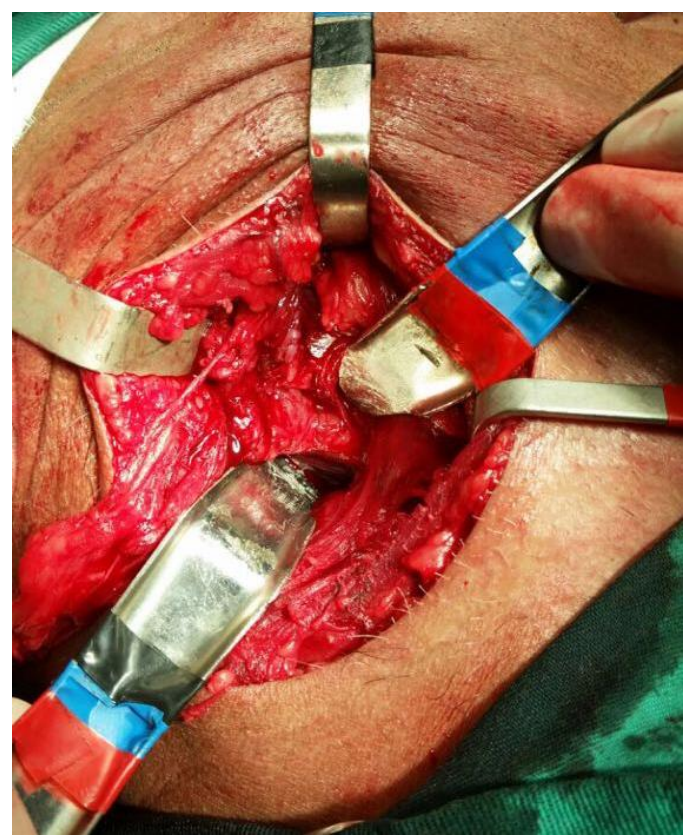

Figura 4: Aspecto clínico após enucleação.

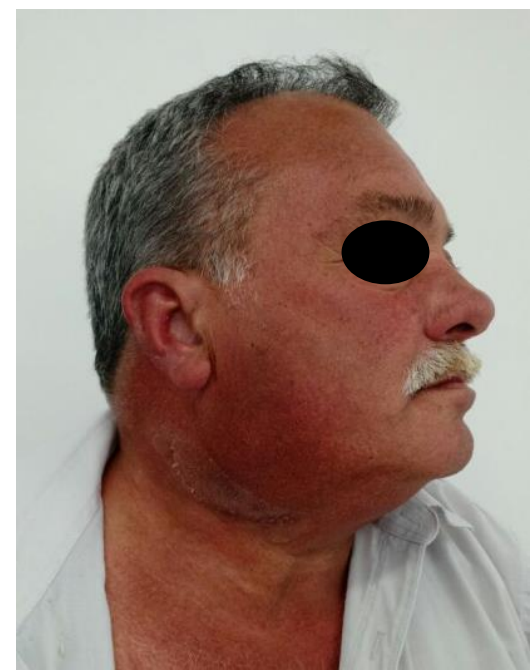

Figura 5: Pós operatório de 3 anos sem intercorrências e recidiva

\section{DISCUSSÃO}

O adenoma pleomórfico é a neoplasia benigna mais comum da glândula parótida, ocorrendo predominantemente no sexo feminino, com uma 
relação em torno de 1,5:1, e com média etária que pode variar de 36 anos a 45,7 anos ${ }^{3}$.

Clinicamente, o adenoma pleomórfico da glândula parótida apresenta-se como lesão nodular única, com margens bem delimitadas, superfície lobulada, consistência endurecida, móvel e indolor à palpação ${ }^{6}$. A grande maioria $(90 \%)$ dos casos acometem o lobo superficial da glândula, sendo $80 \%$ na sua porção inferior ${ }^{3}$. Microscopicamente, a variedade de tipos celulares é a principal característica do adenoma pleomórfico, não apenas entre diferentes tumores mas também em diferentes partes de um mesmo tumor ${ }^{7}$. O adenoma pleomórfico é composto por elementos epiteliais, mioepiteliais e mesenquimais, envoltos em um estroma de natureza mixóide, condróide ou mesmo osteóide ${ }^{7}$. O diagnóstico da neoplasia de parótida é feito a partir da história clínica minuciosa e pelo exame físico. Os exames de imagem, particularmente a ultrassonografia e a tomografia computadorizada, não são essenciais e podem ser realizados em casos selecionados para planejar o tratamento ${ }^{10}$. O tratamento mais utilizado para o adenoma pleomórfico consiste na excisão cirúrgica com margem de segurança ${ }^{11}$. O prognóstico é considerado excelente quando a cirurgia é feita de maneira adequada, com um índice de cura de aproximadamente $95 \%{ }^{12}$.

\section{CONCLUSÃO}

Conclui-se que, a opção cirúrgica por enucleção, é de grande valia no arsenal do cirurgião, principalmente nas lesões que estão localizadas no lobo superficial, e apresentam-se bem delimitadas e circunscritas.

\section{REFERÊNCIAS}

1. Seifert G, Sobin L. Histological typing of salivary gland tumors. World Health Organization. International histological classification of tumors, 2.ed. Berlin: Springer-Verlag; 1991.

2. Spiro RH. Salivary neoplasms: overview of a 35year experience with 2807 patients. Head Neck Surg. 1986; 8(3):177-84

3. Witt RL. The significance of the margin in parotid surgery for pleomorphic adenoma. Laryngoscope 2002; 112(12):2141-54

4. Louro RS, Passeado D, Andrade MC, Sampaio RKPL. Adenoma pleomórfico em palato duro: relato de caso clínico. Rev Bras Odontol. 2002;59(1):25-7.

5. Nogueira AS, Alves APNN, Nogueira RAS, Tavares RN. Adenoma pleomórfico no palato duro: relato de caso clínico. Rev Paul Odontol. 2001;23(1):14-8.

6. Ribeiro-Rotta RF, Cruz Ml, Paiva RR, Mendonça EF, Spini TH, Mendonça AR. O papel da ressonância magnética no diagnóstico do adenoma pleomórfico: revisão da literatura e relato de casos. Rev Bras Otorrinolaringol. 2003;69(5):699707.

7. van der Wal JE, Leverstein $H$, Snow GB, Kraaijenhagen HA, van der Waal I. Parotid gland tumors: histologic reevaluation and reclassification of 478 cases. Head Neck. 1998; 20(3):204-7.

8. Cohen MA. Pleomorphic adenoma of the cheek. Int J Oral Maxillofac Surg. 1986; 15(6):777-9.

9. Tiago RSL, Castro GA, Ricardo LAC, Biihler RB, Fava AS. Adenoma pleomórfico de parótida: aspectos clínicos, diagnósticos e terapêuticos. Rev Bras Otorrinolaringol. 2003;69(4):485-89.

10. Kamal SA, Othman EO. Diagnosis and treatment of parotid tumours. J Laringol Otol. 1997; 111(4):316-21

11. Nogueira AS, Alves APNN, Nogueira RAS, Tavares RN. Adenoma pleomórfico no palato duro: relato de caso clínico. Rev Paul Odontol. 2001;23(1):14-8

12.Neville BW, Damm DD, Allen CM, Bouquot JE. Patologia oral e maxilofacial. 2. ed. Rio de Janeiro: Guanabara Koogan; 2004.

\section{CONFLITO DE INTERESSES}

Os autores declaram não haver conflitos de interesse.

\section{AUTOR PARA CORRESPONDENCIA}

\section{Gabriel Mulinari dos Santos}

gabriel_mulinari@hotmail.com
Submetido em 12/07/2019

Aceito em 22/07/2019 